\title{
Protective Effect of Hsp70 Molecular Chaperone in Polyglutamine Diseases Is Realized by Sequestering of GAPDH
}

\author{
A. V. Kaznacheeva, I. V. Guzhova, and B. A. Margulis \\ Institute of Cytology, Russian Academy of Sciences, St. Petersburg; e-mail:avkazn@list.ru
}

DOI: $10.1134 / \mathrm{S} 199074780903026 \mathrm{X}$

At present, neurodegenerative pathologies are the object of active research. Aggregates made of misfolded, mutant proteins causing neuronal death are the reason of the majority of such diseases. One of these illnesses is the Huntington's disease (HD) that is provoked by expanded repeats of glutamine in $\mathrm{N}$-terminal part of huntingtin. The development of the disease stems from the formation and accumulation of SDSinsoluble protein aggregates in neurons of striatum and cerebral cortex. The aggregates are formed by tissue transglutaminase, which catalyzes the ligation of glutamine residues of mutant huntingtin to lysines of other cellular proteins, such as glyceraldehyde-3-phosphate dehydrogenase (GAPDH). The results of our experiments proved that GAPDH is implicated in the formation of SDS-insoluble aggregates because the factors tightly binding the enzyme reduce both the size and amount of aggregates in the cells over-expressing the gene of mutant huntingtin with Q103 repeat. Conversely, the addition of pure GAPDH to ex vivo system of Q103 aggregate formation significantly increased the amount of aggregating Q103.

To explore the mechanism of anti-aggregate activity of Hsp70 in HD model we generated human neuroblastoma cell line with metal-dependent expression of Hsp70. The elevation of the Hsp70 level lead to the reduction of both number and size of aggregates. These data were confirmed by using ex-vivo system of aggregate formation: the addition of pure Hsp70 elevated the amount of soluble polyglutamine-containing protein. Using the method of immunoprecipitation we showed that Hsp70 was able to bind GAPDH in dose-dependent manner: the more Hsp70, the lesser content of GAPDH in insoluble polyglutamine aggregates and the lower amount of the latter. It is concluded that a novel mechanism exists, through which Hsp70 sequesters GAPDH from the control of transglutaminase and by this diminishes cell mortality from cytotoxicity of the aggregates. 